\title{
Déontologie et éthique dans le débat : une expérience de consultation du public
}

Frédérique Leroux, Jacques Brie, Michel Hortolan et Jean Étienne Bidou

\section{(2) OpenEdition}

Édition électronique

URL : http://journals.openedition.org/ere/2025

DOI : $10.4000 /$ ere.2025

ISSN : 2561-2271

Éditeur

Centr'ERE

\section{Référence électronique}

Frédérique Leroux, Jacques Brie, Michel Hortolan et Jean Étienne Bidou, « Déontologie et éthique dans le débat : une expérience de consultation du public », Éducation relative à l'environnement [En ligne], Volume 8 | 2009, mis en ligne le 20 décembre 2009, consulté le 21 février 2020. URL : http:// journals.openedition.org/ere/2025; DOI : 10.4000/ere.2025 


\title{
Déontologie et éthique dans le débat : une expérience de consultation du public
}

\author{
Frédérique Leroux, Jacques Brie, Michel Hortolan et Jean Étienne Bidou
}

1 Les associations jouent un rôle actif dans l'espace public. Elles y sont engagées avec des buts généralement bien définis, et leurs actions contribuent à la construction de l'opinion publique. Fréquemment, les associations de protection de la nature ou d'éducation à l'environnement peuvent passer contrat avec l'Éducation nationale et, ou les collectivités territoriales sur des actions d'information, de sensibilisation, de formation et d'éducation. Dans ces cas-là, elles doivent respecter une certaine neutralité, qui est d'ailleurs souvent précisée dans le contrat ou acceptée tacitement. Par ailleurs elles sont dépendantes financièrement, non seulement des contributions de leurs adhérents, mais aussi souvent de subsides publics ou privés. Se pose alors la question de la cohérence dans l'action, et d'une façon générale du débat entre l'engagement qui est celui de l'association et le respect de l'impartialité dans la démarche sur laquelle elle a contracté.

2 Ce questionnement serait sans fin si on ne s'attachait pas à décrire les faits dans leur réalité, les échanges qu'ils suscitent, les choix qui sont faits à la lumière des valeurs qui animent ces associations. C'est ce que nous tenterons de faire pour un cas précis : celui de Charente Nature et de son action dans le cadre de l'animation du débat public lors de l'application de la Directive Cadre Européenne sur l'Eau (DCE), contractualisée avec l'agence de l'eau Adour-Garonne. Les questions qui se sont alors posées ont été, d'une part en interne la crainte que la responsabilité d'une animation du débat public se voulant impartiale ne prive l'association de sa liberté de parole militante, et d'autre part avec certains des acteurs des territoires la contestation de l'impartialité de l'association.

3 Après avoir brièvement décrit la situation, on s'attachera à examiner les procédures qui ont été mises en place pour garantir l'impartialité dans l'animation des échanges avant 
d'essayer d'en tirer quelques règles plus générales quant aux différents rôles que peut jouer une association dans l'espace public.

\section{Charente Nature et la mise en place de la DCE - Les arguments du débat interne}

\section{Charente Nature : une association de protection de la nature et de l'environnement}

4 Forte de 500 membres parmi lesquels une dizaine d'associations locales, Charente Nature a été créée en 1970 et a tout de suite adhéré à France Nature Environnement (Fédération nationale des associations de protection de la nature et de l'environnement). Peu après, les quatre associations départementales, dont Charente Nature, ont créé Poitou-Charentes Nature. L'association est membre du Groupe Régional d'Animation et d'Initiation à la Nature et à l'Environnement (GRAINE) et adhère au réseau national École et Nature. Elle participe également au travers de la Coordination Environnement et Développement Durable (CEDD) à la vie d'un réseau dense d'associations ayant en commun l'environnement.

5 Charente Nature est une association généraliste agréée ${ }^{1}$ au titre la protection de la nature, de l'urbanisme et de l'éducation. Elle a pour objectif de concourir à la protection et à la mise en valeur de la nature et de l'environnement en Charente. Un aspect de ses activités est celui d'une association naturaliste: il concerne les inventaires et les expertises portant sur la connaissance du patrimoine naturel. L'association agit en outre dans le domaine de la protection de la nature ${ }^{2}$ et de la qualité de l'environnement. L'éducation des publics est un domaine essentiel de son activité. L'association occupe une équipe de trois animateurs professionnels intervenant surtout en milieu scolaire et se consacre aussi à la formation des adultes. La revue Charente Nature est dédiée à l'information sur l'actualité environnementale. Enfin, Charente Nature conduit une importante activité institutionnelle puis qu'elle siège dans une cinquantaine de commissions administratives de niveau régional, départemental ou local.

6 C'est donc en raison de ses statuts, pour son intérêt dans la qualité de l'environnement et comme acteur dans le jeu institutionnel, qu'elle était concernée par la Directive Cadre sur l'Eau.

\section{La mise en place de la DCE}

7 Le Parlement et le Conseil européens ont adopté le 23 octobre 2000 la Directive Cadre sur l'Eau (DCE), transposée en droit français le 21 avril 2004. La DCE vise à apporter une vision d'ensemble à la politique européenne de gestion de l'eau et à établir un cadre européen pour la protection des eaux continentales, souterraines et côtières. Elle fixe un objectif clair et ambitieux : le bon état écologique des eaux en 2015.

8 La DCE, qui s'inscrit dans l'esprit de la convention d'Aarhus signée en 1998, stipule que le public, c'est-à-dire tous les citoyens en tant qu'usagers de l'eau, devra être largement associé à toutes les étapes de la démarche. La France a donc lancé une première 
consultation du public sur les enjeux de la politique de l'eau, organisée par les agences de l'eau ${ }^{3}$ qui ont un rôle déterminant dans la mise en œuvre de la DCE.

9 Au deuxième semestre 2004, les partenaires institutionnels ${ }^{4}$ sont consultés sur le programme de travail pour la révision du Schéma directeur d'aménagement et de gestion des eaux (SDAGE) $)^{5}$ Selon les documents élaborés par les agences de l'eau, seulement une rivière sur huit et aucune nappe souterraine n'atteindrait le bon état en 2015 si rien ne changeait dans nos pratiques.

10 Les agences de l'eau ont ensuite lancé un appel à projets pour la consultation du public en 2005 portant sur l'état des lieux des ressources en eau élaboré par chacune des instances de bassins et sur le programme de travail devant conduire à la révision des SDAGE. Cet appel à projets était adressé à divers acteurs telles les associations de protection de la nature et de l'environnement, et toute association identifiée comme acteur-relais vers le grand public. Puis, du 2 mai au 2 novembre 2005, ces acteurs, parmi lesquels figurait Charente Nature, sont allés à la rencontre du grand public pour lui expliquer les objectifs de la DCE et l'encourager à exprimer son opinion au moyen des questionnaires rédigés par les six agences de l'eau.

\section{Le débat interne}

11 Les réflexions internes à Charente Nature ont été précédées par des travaux effectués par France Nature Environnement, la Fédération nationale des associations départementales comme Charente Nature ${ }^{6}$. Cependant, la décision de s'engager ou non sur l'appel d'offres des agences de l'eau appartenait aux associations départementales et a fait l'objet d'un débat.

Les arguments contre l'engagement dans cette démarche étaient la crainte de ne pas pouvoir exprimer librement sa position, la crainte d'être manipulé et ainsi de cautionner des organisations dont les objectifs ne sont pas la protection de l'eau. Il fallait en outre considérer les coûts causés par la mobilisation de moyens matériels et humains qui pourraient manquer pour d'autres activités.

Pourtant la majorité a considéré qu'il est dans le rôle de l'association de contribuer à l'information $\mathrm{du}$ public, et d'avoir un rôle constructif dans cette nouvelle réglementation dont elle partage l'esprit. En effet, les associations jouent un rôle de plus en plus affirmé dans l'animation du débat public (Barthélémy, 2000). Elles ont non seulement un rôle d'alerte, mais aussi de questionnement et, par leurs compétences et leur expérience dans les animations et débats, elles sont force de proposition et d'action. Elles servent également de recours, ou au moins de source d'information au sujet d'agissements qui porteraient atteinte à l'environnement (carrières, stockages de déchets, pollutions, gestion de l'eau, aménagements divers, etc.). Les responsables ont donc estimé que l'association, généralement ouverte au dialogue, se trouvait en capacité d'entendre d'autres avis et de favoriser les échanges.

La décision de s'engager a été prise par le Conseil d'Administration de Charente Nature après présentation pour avis à la commission eau de l'association. Par la suite Charente Nature a informé ses associations membres et les partenaires de son projet, qu'elle s'engagerait dans une convention avec l'agence de l'eau Adour-Garonne, qu'elle serait vigilante sur le rôle qu'on pourrait lui faire jouer, et qu'elle rendrait compte du travail effectué. 


\section{La déontologie du débat public}

15 Notre choix de se positionner clairement comme un acteur relais en capacité d'animer ce temps de consultation ayant été validé par les instances de notre association, notre proposition ayant été retenue par l'agence de l'eau Adour-Garonne, il restait à concevoir une méthode susceptible de garantir l'impartialité attendue et nécessaire dans ce type d'exercice. Elle a nécessité de réfléchir sur les principes de l'action et d'élaborer une démarche qui les respecte.

\section{Les principes}

Notre réponse à l'appel à projets de l'agence de l'eau Adour-Garonne était organisée autour de trois entrées :

- être un relais efficace de la consultation,

- apporter les éléments essentiels de connaissance,

- recueillir, à l'occasion de celle-ci, une expression sur les propositions d'actions à mettre en œuvre à partir de l'état des lieux réalisé par l'agence?

Notre choix a d'abord consisté à s'appuyer sur les différentes échelles de territoires de notre département et en particulier sur les «pays ». Dans leur forme actuelle, donnée par la loi dite « Voynet » (Loi d'Orientation pour l'Aménagement et le Développement Durable du Territoire, 1999), ils sont dotés d'un Conseil de Développement où travaillent les acteurs du territoire, et constituent ainsi des laboratoires où la participation des habitants sur des territoires de projets est en cours d'expérimentation.

Dès février 2005, à la suite d'une première rencontre d'information et d'échange sur les modalités d'action à construire ensemble, les travaux ont été conduits spécifiquement avec chacun des Conseils de Développement. Si chacune de ces démarches territorialisées a été vécue et assumée de manière différente, il est possible d'exposer les principes communs :

- Charente Nature est responsable de la logistique générale. L'association se charge de la préparation et de l'édition de supports de communication (en intégrant par un travail partagé avec eux la spécificité de chacun des territoires) et de l'apport de données et d'informations nécessaires à la compréhension des enjeux de la DCE, sous forme d'un diaporama. L'association est également responsable de l'animation de la soirée-débat choisie comme un des temps forts de la démarche.

- Chaque Conseil de Développement, en lien avec les élus et les services de son Pays ou de son Agglomération, assure une recherche de partenariats locaux et une communication visant à une mobilisation du « grand public ».

- L'animation est organisée autour de deux temps; la soirée-débat et une demi-journée sur le terrain organisée avec des acteurs locaux sur une problématique spécifique. 


\section{Neutralité ou impartialité ? La confection des supports de communication} a permis à chacun de se préparer pour la soirée débat. Notre souci, au cours de ce temps de travail avec les acteurs locaux, était que ce support reste au plus près de ses deux objectifs : dresser l'état des lieux et transmettre des apports de connaissances.

\section{Faire se rencontrer les intérêts contradictoires}

Le débat constitue le deuxième temps de la soirée. Comme dans la plupart des débats, la question était celle de la possibilité de faire se rencontrer les tenants d'intérêts contradictoires et de les mettre en situation d'argumenter en évitant le monopole de la parole par quelques ténors en représentation au milieu d'un parterre réduit au silence. Nous avons adopté quelques règles liées à ce que nous concevons comme la déontologie du débat:

- Toute personne participant à cette soirée débat doit pouvoir être mise en situation de s'exprimer et d'argumenter. Elle doit pouvoir le faire dans des conditions favorisant l'écoute de chacun.

- La parole de chacun est chargée de sens, et ce d'autant que les idées exprimées seront reçues dans d'autres lieux.

- Toutes les opinions sont discutables et elles le sont de manière égale. La discussion ne se construit pas sur le modèle raison/tort.

- Face à la nature du débat, le consensus n'est pas recherché. Les extrêmes peuvent s'exprimer et être entendus. 
- Constituer des petits groupes (cinq personnes), chacun se dotant d'un secrétaire chargé de recueillir toutes les propositions. La consigne était d'échanger d'une manière argumentée sur des propositions, sans chercher à se mettre d'accord ni décider qui a tort ou raison, l'écoute des arguments de chaque personne du groupe étant aussi importante que l'expression de ses propres arguments.

- Donner un statut particulier à la production écrite. La personne chargée des prises de notes assurait donc la mémoire de toutes les propositions formulées. Plus tard, la totalité de la production écrite sera saisie, classée selon les thèmes sans autre transformation et transmise intégralement à l'agence de l'eau Adour Garonne, au Conseil Régional PoitouCharentes, au Conseil Général de la Charente, à la Direction Départementale de l'Agriculture et de la Forêt (DDAF). Une réunion ultérieure sera consacrée à la restitution de cette production écrite auprès des partenaires locaux.

- Terminer par le grand débat. À la suite de ce travail en groupe, le troisième temps de notre soirée était organisé dans une configuration plus classique, en réunion plénière. Nous étions simplement attentifs à la gestion du temps afin qu'un maximum de personnes puisse s'exprimer.

Ainsi peut-on résumer le déroulement de nos réunions de préparation sur chacun des territoires. La validation du diaporama et de la méthode d'animation par les acteurs locaux ainsi que le retour des propositions émises pendant les réunions vers ces mêmes acteurs étaient au cœur de notre dispositif.

\section{De la préparation à la réalité vécue}

Des six soirées, nous retenons la qualité des productions écrites et la sérénité du débat final. La qualité d'écoute lors du temps d'échange ne s'est jamais démentie malgré le fait que les intérêts des participants s'opposaient fortement. C'est probablement grâce à ce travail en petits groupes que les prises de parole dans le grand débat se sont exercées calmement. Pour autant, alors que les oppositions s'exprimaient, chacun a pu prendre la mesure des réelles différences de conception. Dans les semaines qui ont suivi cette phase de consultation, nous avons reçu des retours positifs, y compris de la part de structures ayant des positions différentes de notre association.

Malgré tout, la légitimité de Charente Nature à animer ce débat a été attaquée sur deux points essentiels. Comment une association militante peut-elle organiser un débat impartial ? Comment peut-on être sûr que la démarche n'est pas manipulée et sert réellement à quelque chose ? La première est une question fondamentale qui avait été déjà débattue à l'intérieur de l'association et dont cet article tente de rendre raison. La seconde reste ouverte à chaque débat public.

La réalité vécue et perçue de ces réunions nous amène à penser que nous avons créé les conditions de l'impartialité telle que nous l'avions définie au cours de notre réflexion en amont et au cours des rencontres de préparation avec les acteurs locaux. Cette 
perception positive n'évacue pas la claire conscience que nous n'avons pas été perçus comme impartiaux par la totalité des participants.

\section{Débat public et action associative}

31 Mais au-delà de la réflexion sur la conduite du débat public, l'élaboration de règles et de procédures, le souci d'impartialité qui anime toute la démarche, il demeure le problème essentiel : pourquoi avoir choisi cette forme d'action? Pourquoi ne pas être resté dans un rôle plus classique et peut-être plus efficace pour l'association: l'occupation et l'animation de l'espace public pour faire valoir ses arguments sur la gestion de l'eau? C'est d'ailleurs cette question qui avait été débattue au sein de l'association.

On peut évacuer assez rapidement les arguments purement politiques. Charente Nature estimait qu'un débat public impartial, où l'essentiel de l'information provenait des agences de l'eau, et sur laquelle elle était en accord, ne pouvait que faire avancer ses idées. Dans ce contexte favorable, où l'association avait jugé que la DCE allait dans un sens global satisfaisant, il était nécessaire de s'assurer que le débat soit efficace et, précisément, impartial. Cet aspect existe, il est certainement important dans la prise de décision de répondre à l'appel à projets, mais la totalité de la démarche demande à être éclairée à la lumière des valeurs défendues par l'association. La valorisation de l'expression du plus grand nombre de citoyens et ceci en évitant d'offrir une tribune aux seuls usagers porteurs d'intérêts privés économiques forts explique également l'engagement de l'association.

\section{L'engagement associatif dans le débat public}

Ce qui importe dans le débat, c'est que tous les points de vue puissent être exprimés, la volonté générale résulte alors de l'accord sur le meilleur argument dans une discussion transparente et libre. Dans ce jeu où les logiques d'acteurs s'opposent, cristallisées autour d'intérêts contradictoires, nous pensons que participer au débat public et l'alimenter en affirmant des positions est juste.

Ainsi, Charente Nature est au quotidien concernée par des débats sur des questions socialement vives. Nos idées y sont rarement majoritaires. Dans notre contexte démocratique, nous siégeons dans des instances où nos positions peuvent être exprimées, nous éditons "librement» une revue, nous sommes présents dans de nombreuses manifestations publiques, nous sommes interpellés par des acteurs qui nous trouvent trop frileux ou outrageusement partiaux, nous sommes interrogés par des élus qui nous jugent gênants ou pas assez à leurs côtés.

Les modalités de rencontre, d'explication, d'essai de compréhension, d'affrontement, sont multiples. Certaines d'entre elles demandent l'application de règles déontologiques. Pas plus que Sandrine Rui (2002) nous ne pensons que le débat public soit « un lieu neutre où des citoyens vertueux, raisonnables, désintéressés, instruits et compétents discuteraient d'une façon courtoise avec pour seul horizon la production conjointe du bien commun ». Notre association se place comme les autres organisations sur ce terrain. Nous avons des idées que nous cherchons à faire partager et prévaloir au nom de ce que nous pensons bon pour l'intérêt général (Planche, 2007). 

ne pensons plus qu'il soit l'expression commune, issue d'un dépassement des intérêts individuels, où le citoyen actif porteur de convictions et de projets ne peut vouloir que ce qui est bon pour la collectivité. Nous sommes rétifs à un intérêt général qui ne serait que la somme des intérêts particuliers, et où le consommateur, l'entrepreneur, l'individu bâtissent un compromis avec les autres acteurs de la société civile. Nous adhérons plutôt à l'idée que le débat public est un cadre permettant à chacun de construire et d'exposer son point de vue sur un projet. «Il est l'occasion de fonder rationnellement l'utilité publique en mobilisant des données vérifiables, traduisant faits, valeurs et intérêts en objets de connaissance » (Rui, 2002). «Le projet ne sera légitime que si les conditions cognitives pour l'évaluer sont réunies » (Rui, 2002).

C'est effectivement ainsi que nous avons construit le débat sur l'eau : en apportant une information la plus complète possible afin de permettre à chacun de construire son opinion. Nous nous sommes écartés du premier modèle du débat où nous aurions cherché à faire prévaloir nos idées, nos valeurs et nos convictions, pour œuvrer à la réussite du débat en mettant en capacité les participants de mener une discussion. Nous sommes passés d'une fonction «tribunicienne » à une fonction éducative.

\section{Citoyenneté et éducation populaire}

La société civile est aussi le lieu d'apprentissage de la citoyenneté et de la participation politique. Former des citoyens libres et informés qui s'engagent volontairement dans la vie publique est un des buts essentiels de l'éducation populaire. Il s'agit de construire une égalité qui permette à tout le monde d'accéder à l'espace public (Lapeyronnie, 1999). Or, cette égalité, si elle est évidente en droit, ne l'est guère dans les faits. De nombreuses difficultés s'interposent. En particulier, le fonctionnement politique «infériorise insidieusement les uns pour confier aux autres experts «de toutes confessions ", disciplinaires, techniques, idéologiques ou politiques, la prise en main de nos destinées individuelles et collectives » (Dupont-Lourdel, 2001). Or, le niveau des connaissances ne peut être confondu avec les capacités d'analyse et d'invention des groupes en situation.

L'acte de penser ne surgit pas spontanément de la situation, il se construit. Par l'éducation, et particulièrement l'auto-éducation, il devient possible de combattre ce qui empiète sur notre autonomie, individuellement et collectivement, comme se construisent la pratique du doute et l'esprit critique.

Encore faut-il être en capacité de s'exprimer et d'être entendu. Sur ce point, DupontLourdel (2001) fait une intéressante distinction entre ce qu'elle appelle le militantisme socio-politique et le militantisme socio-culturel. Le mouvement socio-politique est le plus commun : le militant ou l'association militante prend position dans l'espace public pour conquérir (pour ses idées) du pouvoir ou au moins de l'influence. «Le mouvement socio-culturel [...] ne se propose pas de conquérir ou d'exercer le pouvoir politique. Il vise à changer à long terme les mentalités par une logique culturelle, informative ou éducative » (Dumazedier, 1995). Agir avec d'autres sur le milieu pour le transformer nécessite l'exercice de son intelligence, le développement de son autonomie et l'entraînement à la prise de responsabilité sous une forme démocratique. C'est ce qui a été tenté lors de la démarche de la soirée débat, où a été particulièrement travaillé le processus de production d'argumentations. Nous nous reconnaissons dans le 
militantisme socio-culturel avec la volonté d'augmenter les capacités d'expression et d'intervention de chaque participant, la valorisation de la diversité des engagements et des points de vue (même lorsqu'ils étaient particulièrement contraires à nos idées, tels les agriculteurs irrigants), un entraînement aux attitudes démocratiques (par exemple en écoutant la parole d'autrui), et l'affirmation du principe politique - et non scientifique - de l'égalité des intelligences (et nous avons pris un soin particulier à envelopper du respect nécessaire toutes les propositions qui ont émergé des groupes).

\section{Conclusion}

Est-ce à dire que, l'opiniâtreté, le conflit, la lutte, le recours au droit, l'appel à l'opinion, la construction des rapports de force, sont des vieilles lunes que notre "modernité " aurait rendues inutiles? Nous ne le pensons pas. Pour que la démocratie soit vivante, il est bon que, dans l'espace public, les extrêmes puissent être repérés par chacun. Ne pas prendre sa place dans ce paysage, c'est disparaître. Nous n'abandonnons pas le projet de diffuser nos idées, de les voir reprises et appliquées avec l'aval d'une majorité des acteurs de la société, mais nous le faisons en alimentant la recherche d'un continuel dépassement de la démocratie, afin qu'elle tienne ses promesses. Ce choix est-il partagé au sein des associations adhérant aux réseaux dont Charente Nature fait partie et par tous les adhérents de notre association? Rien n'est moins sûr. Certains peuvent penser que faire valoir nos idées pour la nature et l'environnement ne nous impose pas la responsabilité de faire vivre la démocratie.

\section{BIBLIOGRAPHIE}

Barthélémy, M. (2000). Associations : un nouvel âge de la participation. Paris : Presses de Science Po.

Dumazedier, J. (1995). L'autoformation en chantier. Éducation permanente, 122, 243-256.

Dupont-Lourdel, E. (2001). Militant culturel, militant politique. La Lettre de Peuple et Culture, 24, 5-10.

Lapeyronnie, D. (1999) Que peut-on entendre aujourd'hui par éducation "populaire » ? Conférence introductive au Séminaire sur l'éducation populaire organisé par la Direction régionale Jeunesse et Sports d'Auvergne, 27 avril 1999, Auvergne.

Planche, J. (2007). Société civile - un acteur historique de la gouvernance. Paris : Éditions Léopold Mayer.

Rui, S. (2002). Le débat public comme exercice de la citoyenneté. Les cahiers du Conseil général des Ponts et Chaussées, 6, 18-21. 


\section{NOTES}

1. En France, l'agrément est la reconnaissance d'une association par les pouvoirs publics, lui donnant ainsi accès à des sièges dans diverses instances, permettant d'agir en justice, d'intervenir en milieu scolaire, par exemple.

2. Par exemple, l'association gère le Centre régional de sauvegarde de la faune sauvage.

3. Les agences de l'eau sont des établissements publics placés sous la tutelle du ministère chargé de l'environnement et sous celle du ministère chargé des finances. Elles partagent le pays en 6 grands bassins et ont pour mission de faciliter les diverses actions d'intérêt commun telles que la préservation et l'amélioration de la ressource en eau, la lutte contre la pollution, la connaissance des milieux, l'information des publics sur l'eau.

4. 4 À savoir les Conseils Régionaux, les Conseils Généraux, les Assemblées consulaires et les Établissements Publics Territoriaux de Bassin (EPTB), élargis aux principales agglomérations, les Conseils Économiques et Sociaux, les Comités régionaux et national de pêche maritime, les Comités régionaux conchylicoles ainsi que les membres des Commissions géographiques.

5. Le SDAGE fixe pour chaque bassin hydrographique les orientations fondamentales d'une gestion équilibrée de la ressource en eau dans l'intérêt général et dans le respect de la loi sur l'eau.

6. Par exemple : la rédaction d'un Guide sur la mise en œuvre de la DCE (janvier 2003) ou la tenue de diverses réunions : 1 et 2 octobre 2004 séminaire en présence des agences de l'eau; réunion nationale du réseau Eau et présentation de la DCE.

7. Compte tenu de ces choix, notre candidature supposait également le recrutement d'une personne à mi-temps chargée de la coordination de l'ensemble de ce projet d'animation de la consultation.

\section{AUTEURS}

\section{FRÉDÉRIQUE LEROUX}

Bénévole active à Charente Nature, elle travaille au Conseil Général de Charente en tant que Chargée de mission Prévention déchets.

\section{JACQUES BRIE}

Vice-président de Charente Nature et Rapporteur de la commission Environnement du CESR (Conseil Économique et Social) Poitou-Charentes.

\section{MICHEL HORTOLAN}

Administrateur de Charente Nature et porte-parole de la Coordination Environnement et Développement Durable Poitou-Charentes au sein de la CPCA (Conférence Permanente des Coordinations Associatives) Poitou-Charentes.

\section{JEAN ÉTIENNE BIDOU}

Géographe de formation, il est maître de conférences à l'Institut Universitaire de Formation des Maîtres de Poitou-Charentes / Université de Poitiers. En partie mis à disposition de l'Ifrée (Institut de formation et de recherche en éducation à l'environnement en Poitou-Charentes) dans 
le cadre d'un partenariat entre ces deux institutions, il y est responsable de la mission recherche en éducation à l'environnement. 